Radiologe 2021 · 61:64-66

https://doi.org/10.1007/s00117-020-00794-z

Angenommen: 14. Dezember 2020

Online publiziert: 8. Januar 2021

(c) Springer Medizin Verlag GmbH, ein Teil von Springer Nature 2021

\section{Redaktion}

F. Bamberg, Freiburg (Leitung)

U. Attenberger, Bonn

M. Eisenblätter, Freiburg

I. Molwitz, Hamburg

M. Notohamiprodjo, München

B. Sigl, Wien

A. A. Tavakoli, Heidelberg

L. Ullrich, München

Durch die anhaltende COVID-19-Pandemie haben digitale Lehrveranstaltungen im Medizinstudium sowie digitale Fort-, Weiterbildungs- und nationale wie internationale Kongressformate für ärztliche Kolleginnen und Kollegen einen fundamentalen Aufschwung erfahren (- Infobox 1). Auch nach der Pandemie werden entsprechend vermehrt digitale Angebote erforderlich sein. Das etablierte Spektrum der digitalen Lehre reicht dabei von Aufzeichnungen klassischer Lehrformate über virtuelle Präsenzformate bis $\mathrm{zu}$ interaktiven, autarken Onlineangeboten. Darüber hinaus bietet die digitale Lehre auch die Chance auf Entwicklung innovativer und kreativer neuer Lehr- und Lernformate.

Die konkrete Umsetzung digitaler Lehre sowie Visionen zur Weiterentwicklung virtueller Lehre werden am Beispiel der radiologischen Lehr- und Lernplattform „conrad“ [1] erläutert.

\section{Grundlegende digitale Lehrmethoden}

Als Basisversion digitaler Lehre wird die Digitalisierung bekannter Lehrformate ohne Verwendung von additiven Materialien verstanden, bspw. durch Aufnahme oder Vertonung von Vorlesungen (VodCasts; [2]). Solche Formate sind jedoch nur eingeschränkt zu aktualisieren und bedingen durch fehlende Interaktivität vor allem passives Lernen.

Isabel Molwitz' · Ahmed Othman · Andreas Brendlin · Saif Afat • Jörg Barkhausen • Sebastian D. Reinartz

' Diagnostische und Interventionelle Radiologie und Nuklearmedizin, Universitätsklinikum HamburgEppendorf, Hamburg, Deutschland

\title{
Digitale Lehre mit, durch und nach COVID-19
}

Im Vergleich zu interaktiven Lehrformaten werden sowohl unmittelbare als auch Langzeitlerneffekte des passiven Lernens als signifikant geringer [3] und die notwendige Vermittlungszeit als länger beschrieben.

Hingegen ermöglichen in Form von Videokonferenzen realisierte Seminare die digitale Interaktion zwischen Dozierenden und Studierenden. Das Maß der Interaktion, das als signifikanter Faktor für die Effektivität einer Lehrmethode gilt [4], ist in Videokonferenzen jedoch multifaktoriell abhängig und nicht garantiert. Überdies ermöglichen Videokonferenzen kein asynchrones Lernen, bei dem die Kommunikation zwischen den Dozierenden und Teilnehmenden - z. B. um Fragen zu stellen - zeitversetzt erfolgt. Mutmaßlich aufgrund größerer zeitlicher Flexibilität werden asynchrone Lehrinhalte allerdings von Lernenden in der ärztlichen Weiterbildung bevorzugt [5].

Die Onlineformate können auch kombiniert werden, indem Präsenzlehre über Videokonferenzen erfolgt und durch Vor- und Nachbereitung in Form von Vodcasts unterstützt wird. Vergleichbar zum Blended Learning bei dem Präsenzlehre durch eLearning ergänzt wird, kann so der positive Einfluss der Interaktivität auf den Lernerfolg in einem kontrolliert vorbereiteten Lernumfeld genutzt werden. Das effizient und zeitlich flexibel im Eigenstudium erlernte
Wissen wird überdies durch den Austausch im Gespräch erneut abgerufen und gefestigt.

\section{Infrastruktur digitaler Lehre in der Radiologie}

Unabhängig vom Lehrformat ist die grundlegende Voraussetzung für digitale Lehre performante IT-Infrastruktur. Hierfür sind fakultätsinterne [6, 7], fakultätsübergreifende [8] und fachspezifische Ansätze [9] beschrieben worden. Insbesondere für die radiologische Lehre ist es beispielsweise notwendig, größere Mengen an Bilddaten sicher abzulegen, reliabel und schnell abzurufen und mit diesen interaktiv zu agieren. Ein Beispiel für eine solche radiologische online Lehr- und lernplattform ist „conrad“. Auf „conrad“ werden Onlineangebote radiologischer Institute deutschlandweit gesammelt und können nach Zielgruppe (Studierende, [Fach-]Ärztinnen/ Ärzte, MTRA), Subspezialität, Format (z.B. Fallsammlung, Zertifizierungskurs, Vortrag) und Publikationsjahr gefiltert abgerufen werden.

So bietet „conrad“ für Studierende ein modulares Kurssystem „unirad“, das im Rahmen der Pandemie ausgebaut wurde und nun nahezu das gesamte radiologische Curriculum im Medizinstudium abdeckt. Die Module basieren auf Vodcasts und realen Fällen, die durch die Beantwortung von Multiple-choice-Fragen 


\section{Infobox 1 Beispiele aktueller digitaler Lehrinhalte in der Radiologie \\ - Digitale Inhalte des European Congress of Radiology 2020 \\ https://www.myesr.org/How_to_watch_ ECR20200nline \\ - Highlightvorträge des Digitalen Röntgen- kongresses 2020 \\ https://academy.mevis.de/drg/courses/ description/403/ \\ - Einführungsvortrag des studentischen modularen Kurssystems auf "conrad" https://academy.mevis.de/drg/courses/ description/395/}

interaktiv bearbeitet werden. Die radiologische Befundung wird dabei durch Vermittlung klinischer Informationen, aktives Scrollen durch die Schnittbilddatensätze, Möglichkeiten zur Optimierung des Bildkontrasts (fenstern) und zur Vergrößerung von Bildausschnitten imitiert. Die Lehrmaterialien und Fälle stammen von fachlich und didaktisch profilierten Kolleginnen und Kollegen verschiedener Standorte, wodurch synergistisch personelle Ressourcen im jeweiligen Institut gespart werden. $\mathrm{Zu}$ sämtlichen Fragen erfolgt eine Ergebnisevaluation und zusätzliches formatives Feedback im Sinne einer Rückmeldung zum Lernprozess und zum korrekten Handlungsprozedere im simulierten Patientenfall, um Engagement und Motivation [10] sowie den Lerneffekt [11] zu verstärken. Tatsächlich gaben in einer Selbstevaluation vor Bearbeitung des interaktiven Onlinemoduls "Muskuloskelettale Radiologie“ (MSK) $70 \%$ der Studierenden an, die Lernziele aus dem Studium zum Thema Arthrose nicht zu erreichen [12]. Nach Bearbeitung betraf dies lediglich noch $10 \%$. Vor Bearbeitung des Moduls schätzten $92 \%$ die Lernziele zu MSK assoziierten Tumoren als nicht erreichbar ein, nach Bearbeitung nur $19 \%$ (ebd.).

Auch für Ärztinnen und Ärzte in Weiterbildung und auf Facharztniveau sind auf „conrad“-Vodcasts wie, z.B. interaktive, fallbasierte Fit-für-den-FacharztKurse vorhanden. Auf Basis der beschriebenen Infrastruktur ist zudem die Entwicklung eines curriculär strukturierten, standortunabhängigen Kurssystems zur
Vorbereitung auf die Facharztprüfung oder für radiologische Zusatzzertifizierungen möglich.

\section{Chancen und Visionen digitaler Lehre}

Zur Weiterentwicklung digitaler Lehrformate und Onlineplattformen auch nach der Pandemie ist es ein vielversprechender Ansatz, das kooperative Lernen [13] zu stärken. So stellt der interkollegiale fachliche Austausch in der Peergruppe in jeder Lebensphase einen wesentlichen Grundpfeiler des individuellen Lernens dar. Die Nutzung dieser zwischenmenschlichen Dynamik im Sinne einer „Virtual Community“ [14] ist das Grundprinzip von Social-Media-Tools, deren Verwendung in der radiologischen Lehre bereits beschrieben wurde [15]. Die Integration von Social-Media-Funktionen in die digitale Lehre, um kooperatives Lernen mit bekannt positivem Effekt auf den Lernerfolg [13] zu fördern, ist entsprechend naheliegend. Als erstes Beispiel für die Umsetzung digitalen kooperativen Lernens können Expertennetzwerke [16] angesehen werden, die in herausfordernden diagnostischen Fällen Hilfestellung innerhalb der Peer-Gruppe ermöglichen.

Weiterhin könnten über digitale Simulationen zukünftig auch Praktika virtuell realisiert werden, in dem im Team (kooperativ) verschiedene Rollen, z. B.im Schockraum, eingenommen werden und reale Fälle unter der Supervision erfahrener Kolleginnen und Kollegen bearbeitet werden. Diagnostische Entscheidungen könnten entsprechend trainiert [17] und ethisch belastende Situationen wie das Überbringen schlechter Nachrichten zunächst in sicherer Umgebung erlebt werden. Speziell in der Radiologie könnten Simulationen mit unterstützenden Hardwarekomponenten wie Virtual-Reality-Brillen und Handschuhen mit Fingersensoren auch zur Planung und praktischen Übung von Interventionen in die digitale Lehre auf jeglichem Niveau integriert werden.

Zusammenfassend ermöglicht die durch die Pandemie beschleunigte Etablierung digitaler Lehre nicht nur die digitale Transformation bestehender Lehrformate, sondern auch die Ent- wicklung innovativer, inhärent digitaler Formate. In der Radiologie bereits existente Online-Lehr- und Lernplattformen gewähren die ressourcensparende, effektive Umsetzung der digitalen Lehre und bieten die notwendige Grundlage zur Weiterentwicklung der virtuellen Lehre sowohl im Medizinstudium als auch in der radiologischen Weiter- und Fortbildung.

\section{Fazit}

1. Die digitale Lehre ist durch die COVID-19-Pandemie deutlich gestärkt worden und reicht von der Digitalisierung bestehender Lehrformate bis zu innovativen, inhärent digitalen Formaten.

2. Die ressourcensparende Umsetzung digitaler Lehre im Medizinstudium sowie in der radiologischen Weiterund Fortbildung ist über deutschlandweite, bereits bestehende OnlineLehr- und Lernplattformen wie „conrad“ möglich.

3. Weiterentwicklungspotenzial der digitalen Lehre besteht in der Stärkung kooperativen Lernens sowie der Verwendung von Simulationen zum Erlernen praktischer Fähigkeiten, z. B. für radiologische Interventionen.

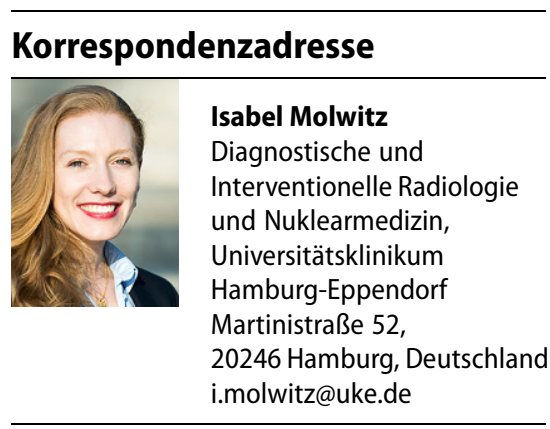

\section{Einhaltung ethischer Richtlinien}

Interessenkonflikt. Die Autoren I. Molwitz, J. Barkhausen, S.D. Reinartz und S. Afat weisen auf ihre Mitgliedschaft in der Vorstandskommission Lehre der Deutschen Röntgengesellschaft e. V. hin. S. Afat, A. Othmann sowie A. Brendlin engagieren sich in den Arbeitsgruppen Co-Rad-19 bzw. unirad für die Erstellung und Pflege des beschriebenen modularen Kurssystems für Studierende. 
Für diesen Beitrag wurden von den Autoren keine Studien an Menschen oder Tieren durchgeführt. Für die aufgeführten Studien gelten die jeweils dort angegebenen ethischen Richtlinien.

\section{Literatur}

1. MeVis Medical Solutions AG Interaktive Lernplattform der Deutschen Röntgengesellschaft. https:// academy.mevis.de/drg/. Zugegriffen: 29. Nov. 2020 (MeVis Medical Solutions AG, Bremen)

2. Backhaus J, Huth K, Entwistle A, Homayounfar K, Koenig S (2019) Digital affinity in medical students influences learning outcome: a cluster analytical design comparing vodcast with traditional lecture. JSurg Educ 76:711-719

3. Subramanian A, Timberlake M, Mittakanti $H$, Lara M, Brandt ML (2012) Novel educational approach for medical students: improved retention rates using interactive medical software compared with traditional lecture-based format. J Surg Educ 69:253-256

4. Offir B, Lev Y, Bezalel R (2008) Surface and deep learning processes in distance education: Synchronous versus asynchronous systems. ComputEduc 51:1172-1183

5. Wittich CM, Agrawal A, Cook DA et al (2017) E-learning in graduate medical education: survey of residency program directors. BMC Med Educ 17:114

6. Back DA, Behringer $F$, Haberstroh $N$, Ehlers JP, Sostmann K, Peters H (2016) Learning management system and e-learning tools: an experience of medical students' usage and expectations. Int J MedEduc 7:267-273

7. Servidio R, Cronin M (2018) PerLE: an "open source", Elearning Moodle-based, platform. A study of university undergraduates' acceptance. Behav Sci 8(7):63

8. Richter-Kuhlmann E (2020) Medizinstudium in Pandemiezeiten: Es geht weiter - trotzdem. Dtsch Arztebl 117(18):A-931/B-786

9. Chang HJ, Symkhampha K, Huh KH et al (2017) The development of a learning management system for dental radiology education: a technical report. Imaging Sci Dent 47:51-55

10. Evans DJ, Zeun P, Stanier RA (2014) Motivating student learning using a formative assessment journey. J Anat 224:296-303

11. Carrillo-de-la-Pena MT, Bailles E, Caseras $X$, Martinez A, Ortet G, Perez J (2009) Formative assessment and academic achievement in pregraduate students of health sciences. Adv Health Sci Educ Theory Pract 14:61-67

12. Othman A, Brendlin A, Afat S (2020) Evaluation des Kurssystems Co-Rad-19

13. Johnson DW, Johnson RT, Stanne MB (2000) Cooperative learning methods: a meta analysis

14. Thoma B, Brazil V, Spurr J et al (2018) Establishing a virtual community of practice in simulation: the value of social media. Simul Healthc 13:124-130

15. Ranginwala S, Towbin AJ (2018) Use of social media in radiology education. J Am Coll Radiol 15:190-200

16. Deutsche Röntgengesellschaft e.V. (2020) Expertennetzwerk Herz- und Gefäßdiagnostik, Muskuloskelettale Radiologie. https://www.drg de/de-DE/3548/expertennetzwerk/.Zugegriffen: 18. Nov. 2020

17. Altabbaa G, Raven AD, Laberge J (2019) A simulation-based approach to training in heuristic clinical decision-making. Diagnosis 6(2):91-99

\title{
Neues Score-System zur Einschätzung des individuellen Risikos für einen schweren Krankheitsverlauf bei COVID-19
}

\author{
Ein neues Score-System zur Einschätzung des individuellen Risikos für einen \\ schweren Verlauf einer COVID-19-Erkrankung haben Wissenschaftlerinnen und \\ Wissenschaftler der Universität Erlangen-Nürnberg und der LMU München \\ vorgestellt.
}

Die Krankheitsverläufe bei einer Infektion mit SARS-CoV-2 sind sehr unterschiedlich und hängen von individuellen Faktoren, wie etwa Vorerkrankungen oder Alter ab. Die Beurteilung des individuellen Risikos für einen schweren Krankheitsverlauf stellt an Ärztinnen und Ärzte somit hohe Anforderungen. Das neue Score-System soll daher eine Hilfestellung zu einer einheitlichen RisikoAbschätzung bieten. In Kenntnis des individuellen Scores kann dann in einem zweiten Schritt beurteilt werden, welche beruflichen Einsatzmöglichkeiten bestehen.

Betriebe und Unternehmen haben eine Fürsorgepflicht für ihre Beschäftigten, insbesondere für jene, die einer Risikogruppe angehören. Eine Beurteilung, ob ein erhöhtes Schutzbedürfnis vorliegt, setzt fundierte Kenntnisse des individuellen Gesundheitszustands sowie der jeweiligen Bedingungen am Arbeitsplatz voraus. Im Rahmen einer Gefährdungsbeurteilung ermitteln die Arbeitgeber mit Unterstützung der Betriebsärztinnen und -ärzte die am Arbeitsplatz objektiv bestehenden gesundheitlichen Belastungsfaktoren. Hierzu zählt auch die Infektionsgefährdung.

Mit dem IKKA-Score haben die Erlanger und Münchner Wissenschaftlerinnen und Wissenschaftler nun ein neues standardisiertes Instrument entwickelt, das die Risikoeinschätzung des Arztes/der Ärztin für einen schweren Krankheitsverlauf bei COVID-19 unterstützt. Das Verfahren wurde in erster Linie für die Anwendung im Arbeitsschutz konzipiert und dient als eine konkrete Entscheidungshilfe im Praxisalltag. Der IKKAScore setzt sich aus den vier Kategorien Immunsuppression, Krankheitsschwere bestehender Vorerkrankungen, Komorbiditäten/Risikofaktoren nach RKI und Alter zusammen und bewertet diese nach einem überschaubaren und leicht verständlichen Punktesystem. Je nach Ausprägung der Risikofaktoren werden in jeder Kategorie Punkte vergeben. Anhand der Gesamtpunktzahl kann dann ein Abgleich mit der sich aus der Gefährdungsbeurteilung ergebenden individuellen Infektionsgefährdung der Beschäftigten erfolgen und risikoadaptierte Einsatzmöglichkeiten für besonders gefährdete Arbeitnehmerinnen und Arbeitnehmer gesucht werden. Die Stärke des neuen IKKA-Scores liegt in der Vereinheitlichung der Risikobeurteilung unter möglichst großer Übersichtlichkeit und geringem Zeitaufwand sowie in der Berücksichtigung bereits vorliegender Empfehlungen des Bundesministeriums für Arbeit und Soziales (BMAS).

Professor Hans Drexler, Direktor des Instituts und der Poliklinik für Arbeits-, Sozial- und Umweltmedizin an der Universität ErlangenNürnberg und Präsident der DGAUM zum IKKA-Score: „Bislang gab es keine einheitliche Vorgehensweise bei der individuellen Risikobestimmung von Arbeitnehmerinnen und Arbeitnehmern. Mit der Entwicklung des IKKA-Scores ist es uns gelungen, Ärztinnen und Ärzten eine Entscheidungshilfe für die Beurteilung der individuellen Gefährdung durch SARS-CoV-2 zur Verfügung zu stellen und somit einen Beitrag zum Schutz von vulnerablen Gruppen am Arbeitsplatz zu leisten."

Eine ausführliche Darstellung des neuen Verfahrens finden Sie hier: https://www.asu-arbeitsmedizin.com/praxis/zur-diskussion-gestelltikka-score-zur-vereinheitlichung-der-beurteilung-des-individuellen

Dr. Thomas Nesseler, Pressekontakt Deutsche Gesellschaft für Arbeitsmedizin und Umweltmedizin e.V. 